\title{
Exploration of Humanistic Design of Hospital Architecture
}

\author{
Humanistic Design of Hospital
}

\author{
$\mathrm{Na}$ Wang \\ Department of anesthesiology \\ The First Hospital of Jilin University \\ Changchun, China
}

\author{
Jinguo Wang* \\ Department of urology \\ The First Hospital of Jilin University \\ Changchun, China \\ *Corresponding author:
}

\begin{abstract}
The hospital is an important place for people to seek medical treatment. The rationality of space design directly affects the psychological comfort of people who seek medical treatment. Modern hospital architecture presents the characteristics of more comprehensive service functions, more various space compositions and more diverse personnel flows. This makes public space and its combination more complicated. The single space mode can no longer adapt to the social development. This article is mainly about the medical space design which should be considered from the physiological and psychological needs of patients. Psychological principles of color and construction should be taken into design to create a comfortable medical environment for patients and help patients to reduce the adverse psychological effect of the environment, for faster recovery.
\end{abstract}

Keywords—humanistic; design; hospital; architecture

\section{INTRODUCTION}

The public space of hospital buildings consists of a number of interrelated and dependent elements, resulting in a complicated and special hierarchical structure, which is the core guarantee of efficient and orderly management of hospitals [1]. Therefore, it needs a strong theoretical framework of the system as a design support, which from macroscopic to microcosmic makes the public spaces as a whole integration of internal and external spaces to meet the users' requirements and to ensure the hospital overall optimization and reasonable extension in the future [2].

Humanization is a kind of concept, which is manifested in the convenience, lifestyle and operation habits of the user, for meeting the needs of the users. Humanization includes humanization management and humanization design. Hospital humanistic design is the core of patient-centered. It is a habit of respecting patients and a kind of humanistic spirit. It is the design of the perfect harmony between men and natures by means of art to show the integration of people and things [3]. Now hospitals are no longer just cold medical devices. Doctors need to focus on the patient's emotional experience and the patient's physical problems. How to alleviate the patient's restlessness in waiting for room and how to guide the depressed mood of the patient in hospital become the content to discuss about the needs of the hospital humanized design.

\section{Systematic DESIGN PRINCIPLES}

The systematic design principle of public space in hospital building should make space elements of the system give full play to the advantages of the integrity of the public space as a hospital central which can guarantee treatment and rehabilitation of pleasure, create the current and lasting value and meet the psychological behavior of different users from basic to advanced needs.

\section{A. Safety Principle}

The safe medical environment system guarantees the hospital to deal with all kinds of emergencies, not only to meet the security. It can fully embody the core idea of humanized medical service.

- Public health security. The outbreak of infectious diseases reminds people that the most important safety problem in hospital public space is to avoid the cross-infection of people. In the design, the function partition should be divided into independent units to avoid unnecessary contacting. In order to reduce the flow distance, reduce the traffic flow density and realize one-way flow, the flow line of different properties is adopted [4].

- Safe evacuation. In the public space of the hospital, the majority of the patients, except for the medical staff, are restricted by different degrees of illness. The particularity of medical architecture decides that its requirement for fire evacuation is more strict, and sufficient security of color preference must be set up to attract many domestic and foreign researchers to explore [5]. These studies use the order of color preference in order to reveal the laws of individual color preference, but the results are different due to the differences between countries, time periods and cultures. Studies have shown that the color habits of Chinese urbanites found that, with the change of time, people have great changes in color preference. 


\section{B. Overall Design Principle}

Systematic design should not be designed for every single space, but follow the principle of system. The functional flow line and structural characteristics of the public space of the whole hospital should be intuitive, simple, clear, clear and easy to grasp and form an integral map of organic integration. Therefore, the public space system will have the overall advantage of every individual irreplaceable space [6].

- Enhancing space recognition. The legibility of the environment refers to the observer's understanding of the space and structure of the building and the ability to identify and to form mental representations and cognitive maps of the environment. After patients or visitors enter the hospital, especially the large medical institution, it is difficult to establish a sense of direction. They often get lost in the large space structure and the public space. Crisscrossing and addressing are difficult. Only in a high degree of recognition of space environment, users will have the environment control, relieve stress and anxiety, move fully with free activities and actively complete a series of medical practice. And it is easy to identify environment also enhances the patient's sense of trust and security in the hospital [7].

- Establishing a space order. In the orderly space, human behavior also presents orderly characteristics. The public space system is not only the static organic whole which is composed of the relevant space elements, but also the dynamic organic whole which is composed of people and activities. The combination order of public space should accord with the patient's medical procedure and attach importance to the different behavior psychology caused by the space environment corresponding to this process. It forms the characteristics of the integration of the space system. First, the space classification settings make the space different. Different function attributes tendency of simplification and clearance. It let consumer be clear at a glance. It uses the differentiation of the crowd and the patient step by step to implement macro order stably and orderly. The level of space division is the microscopic view. Through the deep design of the space elements, it carefully compartmentalizes the spatial hierarchy, to support the user's various activities [8].

\section{MeDiCAl COLOR DESIGN}

\section{A. Color Preference}

The domestic research shows that differences in cultural background will significantly influence on color preference. And it also shows that the differences of gender, age, education degree affect whether color preference has been determined. Compared with the study of color preference, the research on specific color preference is more pertinent and practical. In recent years, studies have found that different presentation methods can influence the color preference of subjects. The color preference of multimedia courseware of college students shows that the colorless is the favorite color of college students. Chinese consumers prefer black, white and blue as the color of clothing, with the least preference for red, yellow and orange. The result of the study on the color preferences of young students on computer operating interfaces is blue which is the most popular background color [9]. Further study finds that college students color preference is different under different emotional states. In calm condition they choose red, yellow, blue, green, white and black. In anger they tend to choose the warm color such as red and yellow. Under fear, they will choose the cold feeling color such as blue, green, white and black.

\section{B. Color Influence}

The color of indoor environment, as a human factor, has a certain influence on people's life and living. It can directly affect people's emotions and the mental health of everyone. With the improvement of people's living standard, the demands of the spiritual world and the environment are becoming more and more demanding. People have become more and more demanding of the hospital environment which has surpassed the only place to cure diseases. White is the primary and traditional color of the hospitals in our country. The wall is white, the doctors and nurses are also in white uniforms. The patient all day look at the cold white, which can let a patient and his family feel depressed, nervous, anxious and depressed [10]. Therefore, it is of great significance and function to construct an inpatient environment that meets the psychological needs of people.

- Shared space. Toll hall and waiting room belong to the sharing space of the hospital, where the personnel is dense and the flow momentum is big. All sorts of people exchange in this space. It is easy to appear noisy and chaos. This type of shared space is large in the overall proportion of the area of medical space. In color, we want to find a color as a overall tone in the design for a hospital, through the choice of the color in which major users be calmed. Large areas face the dominant role of the overall atmosphere of space. The area of the six individuals in the hall space is the big which should choose chroma. The simple and elegant color is favorable in using pure color in large area. The ground can choose the same color, the dark color, which makes the ground and the metope form brightness contrast [11]. The brightness of ceiling color can look high and give a person to open sense. The space is feeling capacious, bright and unbound. The harmonic action of colors can alleviate patient mood effectively and help to improve medical worker efficiency at the same time.

- Transition space. There are some transitional spaces in hospital space, such as corridors, stairwells, etc. The users in these space stay for shorter periods of time. It is often overlooked in design. In the absence of light, this kind of space can easily lead to a 
depressed mood. It can adopt warm tone which makes the space has sunshine feeling [12]. For different corridor floor, it can place different color mark in the local which make the color photograph corresponding to the department. Certain colors are not only helpful for some diseases, but also have guiding function [13]. The department of obstetrics and gynecology can choose the purple line as the mark, because the purple can make women safe. Children can choose yellow, yellow can make children relax. When people see these colors, they can know the department of the floor, the color plays a guiding role.

- Private space. Although the hospital is the public space, there is also a certain private space, such as the clinic and ward. The patient's privacy needs to be protected in this kind of space [14]. Private space needs to pay more attention to patient's emotions in color application. In the previous office design, mostly single white is the main color of the clinic and the doctor's coat was white. White feels pure, quiet and broad, but for large area use it is easy to cause eye strain.

- Different colors show different likes and dislikes. It is often caused by people's life experiences, interests and other factors. The vast blue sky and the boundless sea color are consistent and have a broad open sense. It makes people full of wisdom. Blue around the interior space can make a person quiet and full of infinite daydream. Green is the pronoun of nature and the embodiment of life. Green is full of life and quiet. In green, tension can be alleviating and relaxing [15]. In black, white and opposing all symbols, the white is a good representative to bring people's psychology a clean, pure, clean and expansive feeling. Against the background of it, the other color can appear very bold and bright. Grey is a kind of deep color, which is often used in language to describe the mood of decadence, disappointment and depression. Black is the color of a negative in vision, is considered to be unlucky color and a disaster. It is the color of mourning, a symbol of the loss and vacancies, so black and grey are not favorite color. Colors have a symbolic power that can act on the hearts of people [16]. Therefore, reasonable collocation of cold and warm colors can make the people live in this environment peaceful, comfortable, relaxed and happy. It is beneficial to people's physical and mental health.

\section{CONCLUSION}

The design of hospital building seems to be difficult to get rid of the fixed image characteristics of following function. Because its characteristics are closely related to human life, the design of external forms and internal space also begin to diversify under the powerful control of functions. Especially in the design of public space, the coordination of functions and aesthetics can be well demonstrated. In recent years, the design practice in foreign countries has not only expanded the design thinking and mode of medical architectural space form. It is by drawing on the space design techniques of commercial and hotel construction types. Systematic design of public space should be the organic combination of functional use, mental experience and spatial experience. It is a balance of all kinds of requirements, rich expressive force and appeal of public space. It improves the quality of hospital environment and cultural connotation, makes people more to enjoy pleasure and conveys the human nature.

Medical architecture has strict functional requirements and spatial order. It is a type of building that emphasizes rational thinking. In recent years, in the exploration of foreign concepts and practices, the domestic medical architectural design field has achieved remarkable achievements. However, with the reform of social medical and health system, the change of management model and the concept of social health, the hospital has gradually transited to set the composite function such as medical treatment, prevention or health care.

\section{REFERENCES}

[1] Making London Better for All Children and Young People, A Summary. Greater London Authority. 2007

[2] Finding Lost Space. R. Trancik. 2009

[3] Innovation In Play Environment. Paul F Wilkinson. 1998

[4] Urban Planning Issues to Make a Children-suitable City. Forestry Studies in China. 2009

[5] A Visual Approach to Park Design. Albert J Rutiedge. 2001

[6] Tomorrow of Our City. K Messier. 2009

[7] Design of Children's Play Environments. Senda. 2008

[8] Play and Playscapes. Joe L Frost. 2010

[9] Public Urban Wilderness, Habitat Creation and Enhancement Projects. Tommy Thompson Park. 2008

[10] Design Guide - The design of community hospital. 1991

[11] Health Building Notes. 1995

[12] Appendices Engineering Room layouts Fire safety. 2001

[13] Building Type Basics for Healthcare Facilties. 2000

[14] Hospital. Hoenack August. Time-Saver Standards for Building Types. 2001

[15] Design Guide-The design of hospital main entrance. NHS Estates. 1993

[16] Facilities for cancer care centres. NHS Estates. 2001 